Research Article

\title{
Uncovering the Mechanism of Curcuma in the Treatment of Ulcerative Colitis Based on Network Pharmacology, Molecular Docking Technology, and Experiment Verification
}

\author{
Suxian Liu $\mathbb{D}^{1,2}$ Qiaodong Li ${ }^{10},{ }^{1,2}$ Fengzhi Liu, ${ }^{3,4}$ Hui Cao, ${ }^{1,2}$ Jun Liu, ${ }^{1,2}$ Jingyi Shan, \\ Wenchao Dan $\left(\mathbb{1},{ }^{4,5}\right.$ Jianye Yuan $(\mathbb{1}),{ }^{1,2}$ and Jiang Lin $\mathbb{1}^{1,2}$ \\ ${ }^{1}$ Longhua Affiliated Hospital of Shanghai University of Traditional Chinese Medicine, Shanghai 200032, China \\ ${ }^{2}$ Department of Gastroenterology, China-Canada Center of Research for Digestive Diseases, Institute of Digestive Diseases, \\ Longhua Hospital, Shanghai University of Traditional Chinese Medicine, Shanghai 200032, China \\ ${ }^{3}$ Key Laboratory of Chinese Internal Medicine of Ministry of Education and Beijing, Dongzhimen Hospital, \\ Beijing University of Chinese Medicine, Beijing 100700, China \\ ${ }^{4}$ Beijing University of Chinese Medicine, Beijing 100029, China \\ ${ }^{5}$ Department of Cardiology, China Academy of Chinese Medical Sciences Guanganmen Hospital, Beijing 100053, China
}

Correspondence should be addressed to Jianye Yuan; yjy13842064229@163.com and Jiang Lin; sixteen_liu@163.com

Received 10 December 2020; Revised 22 April 2021; Accepted 31 May 2021; Published 17 June 2021

Academic Editor: Guang Chen

Copyright $(\odot 2021$ Suxian Liu et al. This is an open access article distributed under the Creative Commons Attribution License, which permits unrestricted use, distribution, and reproduction in any medium, provided the original work is properly cited.

Aim. The incidence of ulcerative colitis (UC) is increasing steadily in developed countries, it is plaguing nearly 1 million people in the United States and European countries, while developing countries have had a rapidly increased incidence over the past decades. Curcuma is widely used in treating malaria, UC, Crohn's disease, and colon cancer, which lead to diarrhea and bloody stool. However, the systemic mechanism of curcuma in treating UC is still unclear. Our work was supposed to expound how does curcuma alleviate UC in a comprehensive and systematic way by network pharmacology, molecular docking, and experiment verification. Methods. Traditional Chinese Medicine System Pharmacology Database (TCMSP), Shanghai Chemistry \& Chemical Industry Data Platform (SGST), and papers published in Chinese Network Knowledge Infrastructure (CNKI) and PubMed were used to collect the chemical constituents of curcuma based on ADME (absorption, distribution, metabolism, and excretion). And effective targets were predicted by Swiss Target Prediction to establish the curcuma-related database. The disease targets of UC were screened by GeneCards and DrugBank databases, and Wayne (Venn) analysis was carried out with curcuma targets to determine the intersection targets. AutoDock software and TCMNPAS system were used to dock the core chemical components of curcuma with key UC targets. Protein interaction (PPI) network was constructed based on the STRING database and Cytoscape software. Gene function GO analysis and KEGG pathway enrichment analysis were carried out by using Metascape database. Finally, HE staining was performed to identify the inflammatory infiltration and expression difference in TNF- $\alpha$ and STAT3 before and after the treatment of curcuma which was verified by immunoblotting. Results. Twelve active components containing 148 target genes were selected from curcuma. Potential therapeutic targets of curcuma in the treatment of UC were acquired from 54 overlapped targets from UC and curcuma. Molecular docking was used to filter the exact 24 core proteins interacting with compounds whose docking energy is lower than -5.5 and stronger than that of 5-aminosalicylic acid (5-ASA). GO and KEGG analyses showed that these targets were highly correlated with EGFR tyrosine kinase inhibitor resistance, PI3K-Akt signaling pathway, JAK-STAT signaling pathway, MAPK signaling pathway, and inflammatory bowel disease (IBD). Experiments verified curcuma relieved pathological manifestation and decreased the expression of TNF- $\alpha$ and STAT3. Conclusion. Curcuma relieved the colon inflammation of ulcerative colitis via inactivating TNF pathway, inflammatory bowel disease pathway, and epithelial cell signaling in Helicobacter pylori infection pathway, probably by binding to STAT3 and TNF- $\alpha$. 


\section{Background}

UC is characterized by abdominal pain, diarrhea, and bloody stool [1]. Globally, the annual incidence of UC is about 9-20/ 100,000 and the prevalence is $156-291 / 100,000$ [2]. However, the exact pathogenesis is still not fully clear for the etiology is prototypically diverse. It is known that many factors are involved in the development of UC, interacting environmental, genomic, microbial, and immunological elements [3]. With the deterioration of UC, it will eventually lead to colorectal cancer. Therefore, to prevent before the occurrence of UC, to avoid the complications of UC, and to prevent the recurrence of $\mathrm{UC}$ after recovery, a new treatment is urgently needed.

TCM is gaining its popularity in the ameliorating subhealth state and treating disease. Much more attention has been focused on the treatment of UC by Chinese herb; curcuma is one of the popular ones. In ancient China, curcuma was widely used to invigorating the circulation of blood in clinical applications; nowadays, the function of the positive regulation of inflammatory cytokines in inflammatory diseases [4] and its safety [5] attracted many more clinical trials and experimental verifications.

It is well known that TCM is guided by the theory of TCM and characteristics of being multicomponents, multitargets, and multipathways in the treatment of diseases, which meets the requirements of systematically tackling complex diseases such as colorectal cancer. Guo et al. established the model of colorectal cancer and predicted the traditional Chinese medicine components of inhibiting inflammation-induced tumorigenesis by using network pharmacology method [6]. Gupta et al. gathered curcumin's pleiotropic activities from many research studies to conclude its ability to modulate numerous signaling molecules such as proinflammatory cytokines, apoptotic proteins, cyclooxygenases, and C-reactive protein in human participants [7]. To improve the "one target and one drug" mode to "network targeting multicomponent" mode and to discover traditional Chinese medicine from the perspective of system and molecular level [8], a systemic overall approach of curcuma in the treatment of UC is still needed to verify the previous results and broaden the mechanism of curcuma in the treatment of UC.

\section{Methods}

\subsection{Network Pharmacology}

2.1.1. Active Compound Screening. Traditional Chinese Medicine System Pharmacology Database [9] (TCMSP, http://lsp.nwu.edu.cn/tcmsp.php), Shanghai Chemistry \& Chemical Industry Data Platform (SGST, http://www. organchem.csdb.cn), and papers published in Chinese Network Knowledge Infrastructure (CNKI, https://www. cnki.net/) and PubMed were used to collect the chemical constituents of curcuma. We screened curcuma compounds based on absorption, distribution, metabolism, and excretion (ADME) [10], and pharmacokinetic information retrieval filters were used to retrieve bioactive compounds for further analysis under the conditions of $\mathrm{OB} \geq 30 \%$ and $\mathrm{DL} \geq 0.18$ in TCMSP [9]. We further screen the active ingredients by their effects on the human body. However, the compounds were searched from CNKI, PubMed, and SGST without ADME parameters, so we obtained chemical formula of those components from PubChem (https:// pubchem.ncbi.nlm.nih.gov/) to finish Swiss ADME prediction [11], which was requested that $\mathrm{OB}$ degree was equal to $\mathrm{HIGH}$ and at least two terms of druglikeness were YES [12].

2.1.2. Screening of Possible Targets for Curcuma. PubChem was used to search the chemical structures of the active compounds. Potential targets of curcuma were predicted by Swiss Target Prediction (STP, http://www. swisstargetprediction.) [11]. Probability was used to balance the connection between compounds and targets, which was closer to 1 , and it was more connective. We screened targets by the median of probability to establish potential target database related to curcuma.

2.1.3. Predicting the Possible Targets of UC. Data of UCassociated target genes were gathered from GeneCards (https://www.genecards.org/) [13] and DrugBank [14] (https://www.drugbank.ca/) with the keyword "ulcerative colitis." In addition, articles published in CNKI and PubMed about the known targets of its active compounds were counted [15]. Genes from GeneCards were provided with scores, and genes were selected as UC-related ones whose scores were above the median degree [16].

2.1.4. Gathering Compound-Disease Overlapped Targets. The screened curcuma targets and UC targets were imported into Bioinformatics [17] (http://www.bioinformatics.com. $\mathrm{cn} /$ ), and the overlapped targets of compound-disease were obtained as the potential targets for further analysis.

2.1.5. PPI Network of Compound-Disease Overlapped Targets. Protein-protein interaction (PPI) network was derived based on the STRING database (https://string-db.org/), which covered almost all functional interactions between the expressed proteins [18]. Species were set as "Homo sapiens," and the target interaction information was obtained according to the results of analysis.

2.1.6. Gene Ontology (GO) and KEGG Pathway Enrichment Analysis. The biological process (BP), molecular function (MF), cell component (CC), and Kyoto Encyclopedia of Genes and Genomes (KEGG) pathway enrichment analysis were carried out using Metascape system (https://metascape. org/) [19]. In this research, GO functional annotation and KEGG pathway enrichment analyses were performed using the $P$ value less than 0.05 .

2.1.7. Construction of Active Component-Target-Pathway Network. A visual network was constructed through 
Cytoscape software to reflect the complex relationship between active compounds, filtrated targets [17], and pathways based on KEGG pathway enrichment analysis to reflect the relationship between top pathways, included targets, and active compounds. Nodes represented the compounds, targets, and pathways, while edges indicated the interactions between pathways, targets, and components potentially included in the treatment of UC by curcuma.

2.2. Molecular Docking. Using TCMNPAS system [20] and AutoDock [21] software, the docking energy between the overlapped proteins and chemical ingredients of curcuma was calculated. Between the component and the target by docking score value the binding activity should meet two standards: bind tighter than 5-ASA and binding energy was lower than -5.5 to further filtrate the targets related to the treatment of UC by curcuma.

\subsection{Experiment Verification}

2.3.1. Drugs and Reagents. Curcuma (TCM Pharmacy of Longhua Hospital of Shanghai University of Traditional Chinese Medicine), DSS (MP Biomedicals, USA), absolute ethyl alcohol, Tween-20, xylene substitute (Sinopharm Group Chemical Reagent Co. Ltd.), RIPA Lysis buffer, PMSF, BSA, BCA Protein Quantitation Kit (Beyotime), PAGE gel rapid preparation kit, Multicolor Restrained Protein Ladder (Shanghai EpiZyme Biotechnology Co., Ltd.), $\beta$-actin, anti-STAT3 antibody, anti-TNF- $\alpha$ antibody (Abcam Company, England), HE dyeing (Shanghai Yixin Biotechnology Co., Ltd.), and neutral gum (Shanghai Yiyang Instrument Co., Ltd.) were used.

2.3.2. Consumables. Homogenized tube, ceramic beads, frozen storage tube (Shanghai Yike Biotechnology Co., Ltd.), centrifuge tube (Axygen Company, USA), PVDF membrane (Millipore Company, USA), and 96-well plate (Eppendorf Life Sciences Corporation) were used.

2.3.3. Instruments. H2050r high-speed refrigerated centrifuge (Hunan Xiangyi Company), MIX-S vortex mixer, shaker oscillator (Shiloh, USA), TGear mini centrifuge (Tiangen), heating magnetic agitator (Dalong, Beijing), SIMF140 ice maker (Sanyo, Japan), electronic balance (Sartorius, Germany), enzyme labeling instrument (BioTek, USA), tissue grinding homogenizer (MP Biomedicals, USA), electric constant temperature blast drying oven (Jinghong, Shanghai), electrophoretic system, transfer system, glue rack, ultralow-temperature freezer (SANYO, Japan), microtome (Laika, Germany), and TKY-BMB, electrothermostatic water bath (Hualida).

2.3.4. Animals. Healthy male Sprague Dawley (SD) rats, weighing $180 \pm 20 \mathrm{~g}$, were provided by Charles River Experiment Technology Co., Ltd., and the certificate number is SCXK (Hu) 2017-0005. The rats were housed in the animal room of Shanghai University of Traditional Chinese Medicine.

2.3.5. Preparation of Curcuma. The native herb was selected, and standard decoction pieces were prepared with reference to the Chinese Pharmacopoeia (2015 edition). Extract was prepared by boiling the samples in 8 times amount of water for $30 \mathrm{~min}$. The procedure was repeated 3 times.

2.3.6. Groups and the Construction of the UC Model. SD rats were accepted to the laboratory for 7 days before the experiments. According to the random number table, the rats were divided into 3 groups of 4 rats each: control, model, and curcuma. Except for the control group, the UC model was prepared with 5\% DSS, and the intervention was given according to the group after 7 days.

2.3.7. Drug Administration. The curcuma group was administered continuously by gavage with $2 \mathrm{ml} 0.1 \mathrm{~g} / \mathrm{ml}$ curcuma suspension for 7 days. The other two groups were given saline $1.08 \mathrm{~g} / \mathrm{kg}$.

2.3.8. HE Staining and Western Blot. The steps to stain the samples are as follows: xylene I and xylene II, $10 \mathrm{~min}$ for each; $100 \%$ alcohol I, 100\% alcohol II, 95\% alcohol, and 85\% alcohol, $5 \mathrm{~min}$ for each; water washing for $20 \mathrm{~s}$, hematoxylin for $7 \mathrm{~min}$, water washing for $1 \mathrm{~min}, 1 \%$ hydrochloric acid alcohol for $10 \mathrm{~s}, 50^{\circ} \mathrm{C}$ water washing for $5 \mathrm{~min}$, eosin stain for 2 min, and water washing for 10 s; $85 \%$ alcohol, 95\% alcohol, $100 \%$ alcohol II, and $100 \%$ alcohol I, each for $2 \mathrm{~min}$; and xylene II and xylene I, 3 min for each. Neutral balsam was added after xylene was passerillaged.

For protein extraction, tissues were placed in homogenized tubes; 5 porcelain beads, $500 \mu \mathrm{L}$ of RIPA, and $5 \mu \mathrm{L}$ PMSF were added to each tube. After five times' homogenization, the colon tissue was basically broken. The supernatant was extracted after centrifugation to test the protein concentration and to collocate protein solution. Equivalent amounts of protein $(200 \mu \mathrm{l})$ were denatured at $98^{\circ} \mathrm{C}$ for $10 \mathrm{~min}$ in sample loading buffer, then separated by electrophoresis in $15 \%$ gel, and electrotransferred onto $0.45 \mu \mathrm{m}$ polyvinylidene difluoride membranes for $60 \mathrm{~min}$ at $350 \mathrm{~mA}$. Subsequently, the membranes were blocked in blocking buffer (0.01 M phosphate-buffered saline, 0.05\% Tween-20 with $5 \%$ skim milk) at $25 \pm 5^{\circ} \mathrm{C}$, followed by incubation with primary antibodies against STAT3 $(1: 5000)$ and TNF- $\alpha(1$ : $5000)$ at $4^{\circ} \mathrm{C}$ overnight. After being washed with Trisbuffered saline containing Tween-20 (TBST) for 5 min three times, the membranes were incubated with a horseradish peroxidase-conjugated secondary antibody for $1 \mathrm{~h}$ at room temperature. After the membranes were washed three times in TBST for $20 \mathrm{~min}$ each time, the bands were visualized on $\mathrm{X}$-ray film using an enhanced chemiluminescence western blotting (WB) detection system. The Image $\mathrm{Lab}^{\mathrm{TM}}$ software was used for quantitative analysis. 


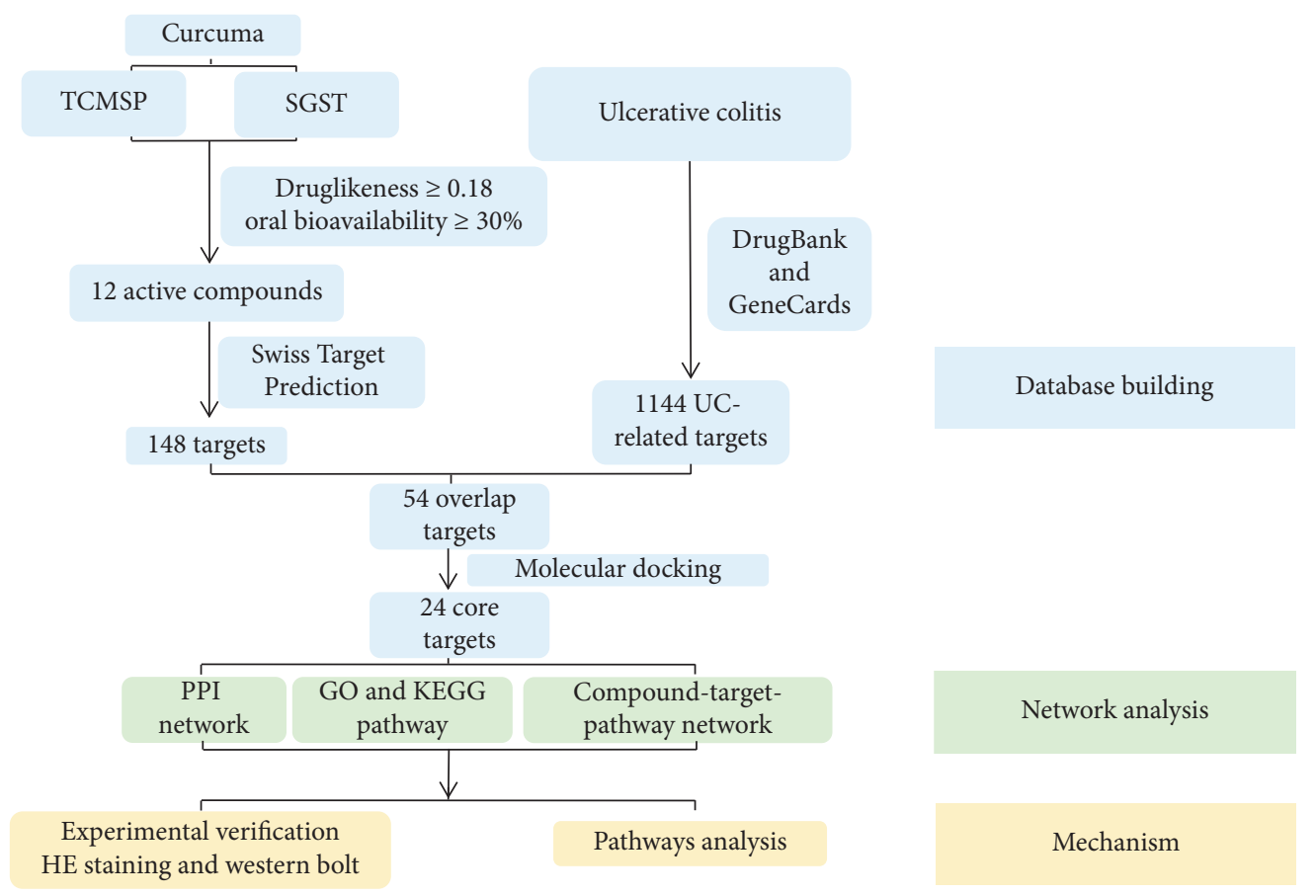

Figure 1: The workflow of the study. Chemical constituents of curcuma were collected from TCMSP, SGST, CNKI, and PubMed based on druglikeness and oral bioavailability. The active compounds were collected further and imported into Swiss Target Prediction to get protein targets of curcuma. Then, we obtained UC-related genes from GeneCards and DrugBank, and coincident genes from curcuma and UC were collected for molecular docking to filter the proteins binding with compounds stronger than 5-ASA. PPI network was carried out by using the STRING database. GO and KEGG pathway analyses were performed by Metascape, and compound-target-pathway network was executed by Cytoscape. Further experimental verification and pathway analysis were carried out to interpret the mechanism.

\subsubsection{The Whole Workflow of Network Pharmacology} Strategy. The workflow of this study is summarized in Figure 1. We built the ingredient-target collection of curcuma and UC-related genes, respectively. The overlapping targets of curcuma and UC were subsequently identified using molecular docking. And the PPI network was constructed according to the targets. Further, the gene ontology (GO) and the Kyoto encyclopedia of genes and genomes (KEGG) pathway enrichment analyses were performed and also compound-target-pathway network was executed. Next, we performed animal experiments to verify the remission of UC by admitting curcuma through HE staining and immunoblotting. Last, key pathways were analyzed to elucidate the mechanism of curcuma in the treatment of UC.

\section{Results}

3.1. Active Compounds and Targets of Curcuma. TCMSP database, SGST, and articles published in CNKI and PubMed were used to gather the active components and targets, and 13 potential ingredients were discovered from curcuma (Table 1). We excluded 1,7-bis(4-hydroxy-3methoxyphenyl)-1,4,6-heptadiene-4-one and determined that 12 active components out of 13 can produce platelet aggregation and other functions in the human body, through literature review [24, 25]. Furthermore, 148 targets were affirmed through PubChem and Swiss Target Prediction.
3.2. Searching the Potential Disease Targets. We obtained the curcuma-related targets from GeneCards (whose relevance score is above the median point) and the ones from DrugBank, CNKI, and PubMed (Table S3). Then the searched results were overlapped with UC targets to obtain the UC-related disease protein targets (Figure 2). Fifty-four potential targets were obtained based on the degree of correlation between curcuma and UC, and their detailed information is shown in Table 2.

3.3. Molecular Docking. We input 54 potential genes into the PDB database (http://www.rcsb.org/) to query their PDB ID [26]. Then, we downloaded the molecular structure of the 12 effective compounds of curcuma in the treatment of UC in ZINC database [27] (http://zinc.docking.org/). Molecular docking was progressed in TCMNPAS to calculate the docking score. The interaction strength between compounds and gene corresponding proteins can be expressed by docking fraction, and the lower the score is, the higher the interaction intensity is. Further, we docked the 54 potential targets with 5-ASA. As shown in Figure 3, the 24 proteins whose docking energy with curcuma compounds matched two standards, the docking energy was above that of 5-ASA and lower than -5.5 , were picked for further network analysis.

3.4. Establishment of PPI Network. The molecular docking filtered 24 core targets for curcuma in the treatment of UC. 
TABLE 1: Basic information for curcuma compound ingredients.

\begin{tabular}{|c|c|c|c|c|}
\hline CAS & Molecule name & Structure & $\begin{array}{l}\text { OB }(\%) / G I \\
\text { absorption }\end{array}$ & Druglikeness \\
\hline $474-62-4^{*}$ & Campesterol & & 37.58 & 0.71 \\
\hline $57-88-5^{*}$ & CLR & & 37.87 & 0.68 \\
\hline $83-48-7^{*}$ & Stigmasterol & & 43.87 & 0.76 \\
\hline $76474-56-1$ & Dihydrocurcumin & & High & 0.55 \\
\hline $22608-12-4^{\boldsymbol{\Lambda}}$ & Bisdemethoxycurcumin & & High & 0.55 \\
\hline $22608-11-3^{\wedge}$ & Demethoxycurcumin & & High & 0.55 \\
\hline $458-37-7^{\wedge}$ & Curcumin & & High & 0.55 \\
\hline $87440-60-6^{\boldsymbol{\Delta}}$ & Curlone & & High & 0.55 \\
\hline $21698-40-8^{\wedge}$ & Procurcumenol & & High & 0.55 \\
\hline $83-46-5^{\wedge}$ & Beta-sitosterol & & High & 0.55 \\
\hline $2309-07-1^{\text {光 }}$ & Methyl ferulate [22] & & High & 0.55 \\
\hline $121-33-5^{54}$ & Vanillin [22] & & High & 0.55 \\
\hline $\mathrm{NA}^{\text {is }}$ & $\begin{array}{l}\text { 1,7-Bis(4-hydroxy-3-methoxyphenyl)-1,4,6- } \\
\text { heptadiene-4-one [23] }\end{array}$ & & High & 0.55 \\
\hline
\end{tabular}

*Ingredients searched from TCMSP. ${ }^{\wedge}$ Ingredients searched from SGST. ${ }^{\star 2}$ Ingredients searched from CNKI and PubMed. 


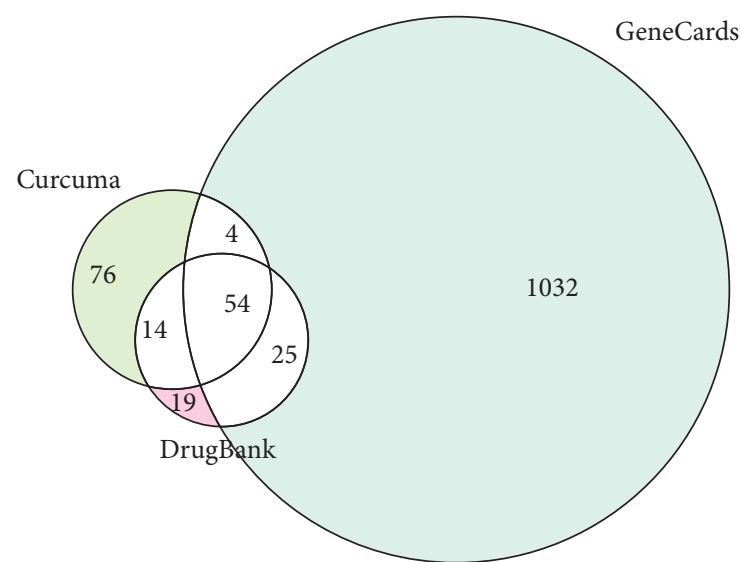

FIGURE 2: Matching of target genes between UC and curcuma.

Then, the 24 genes were uploaded into the STRING database for analysis. We selected protein targets with a medium confidence score of 0.400 and the selected protein targets were plotted as an interaction network. The network of protein-protein interactions (PPI) was established through the STRING database. As shown in Figure 4, 47 nodes and 274 edges were contained in the network; in detail, the average node degree is 12.5 . Nodes represented the core targets and the extended targets, edges represented the connection between the genes, and the degree value represented the association intensity.

\subsection{Gene Ontology Enrichment Analysis and KEGG Pathway} Enrichment. We imported the selected potential 25 target genes into the Metascape system for GO and KEGG pathway enrichment analyses. The results revealed that the functions of these potential targets were related to many biological processes, molecular functions, cellular components, and pathways, which were of crucial importance in the development and treatment of UC. A total of 886 biological processes were enriched based on $P<0.05$, such as cellular response to lipid, response to oxygen levels, and regulation of inflammatory response (Figure 5(a)). A total of 58 molecular function GO terms were enriched (Figure 5(b)). These targets of molecular function mainly involved nuclear receptor activity, transcription factor activity, steroid hormone receptor activity, and many genes related to the molecular functions described above. In all, 18 cell component GO terms were enriched (Figure 5(c)). The targets were closely related to RNA polymerase II transcription factor complex, transcription factor complex, nuclear transcription factor complex, and membrane raft, and many targets were ranked highly as potential related genes. The biological processes, molecular functions, cellular components, and pathways revealed the vital role of curcuma in the treatment of UC.

To further reveal the potential mechanism of curcuma on the effect of UC, we conducted KEGG pathway enrichment analysis on 24 targets and screened out 95 pathways based on the threshold of $P<0.05$ (Figure 5(d)). Numerous pathways for potential target genes were identified, such as epithelial cell signaling in Helicobacter pylori infection related to misregulation of intestinal flora. TNF signaling pathways and inflammatory bowel disease (IBD) are closely related to the inflammatory reaction process. p53 signaling pathway is included in the pathways in cancer. Moreover, MAPK signaling pathway and TGF-beta signaling pathway play a crucial role in immunological stress. In addition, we found some other pathways such as endocrine resistance, hepatitis $\mathrm{B}$, serotonergic synapse, and longevity regulating pathway, which revealed that curcuma has a potential application in other related diseases. The KEGG pathways verified that curcuma cured UC by regulating gut microbiota, inflammatory process, immunization, and inflammatory reaction.

3.6. Component-Target-Pathway Network. To view the relationship between the components, common targets, and their corresponding pathways, a target-pathway network was constructed (Figure 6). Twenty pathways, 47 core common targets, and 12 active compounds were connected. The network contained 88 nodes and 628 edges, in which the green colored circles represented the core common targets, the compounds colored in blue were pathways that contain the targets, and yellow nodes were effective components in curcuma. The pathways with more targets were pathways in cancer, EGFR tyrosine kinase inhibitor resistance, endocrine resistance, and HIF-1 signaling pathway, which correspond to $12,8,8$, and 8 targets, respectively. The result suggested that these four pathways probably played significant therapeutic roles.

3.7. HE Staining and the Effect of Curcuma on the Expression of Key Protein in the Colon of UC Rats. According to the HE staining (Figure 7), DSS could change the crypt structure and inflammatory infiltration in the model rats, which were characterized by the distortion and branch of crypt structures, loss of goblet cells, appearance of a large number of crypt abscesses, lymphocytes, and plasmacyte. The above results indicated that curcuma could ameliorate DSS-induced UC in terms of colon histopathological changes.

Compared with the control group, the protein expression of STAT3 and TNF- $\alpha$ in the colon samples of the model group was significantly increased $(P<0.01)$. Compared with the model group, the curcuma group can reduce the expression of STAT3 and TNF- $\alpha(P<0.05)$ (Figure 8).

\section{Discussion}

UC is one of the autoimmune diseases affecting northern Europe, Canada, and Australia [28]. The occurrence and development of UC are related to commensal microflora, antigen recognition, dysregulation of immunological responses, leucocyte recruitment, and genetic factors [29]. A large number of murine experiments and clinical tests have been performed to identify the bright prospects for the treatment of UC by curcuma, and TNF- $\alpha$ and STAT3 are two research hotspots. It was reported that curcumin and semibionic extraction of compound turmeric can inhibit the proinflammatory signaling by STAT3 and TNF- $\alpha$ in 
TABLE 2: Information on potential targets and the topological attributes.

\begin{tabular}{|c|c|c|c|c|}
\hline No. & Gene name & Protein name & UniProt ID & Degree \\
\hline 1 & AKT1 & AKT serine/threonine kinase 1 & P31749 & 43 \\
\hline 2 & EGFR & Epidermal growth factor receptor & P00533 & 35 \\
\hline 3 & TNF & Tumor necrosis factor & P01375 & 35 \\
\hline 4 & STAT3 & Signal transducer and activator of transcription 3 & P40763 & 33 \\
\hline 5 & PTGS2 & Prostaglandin-endoperoxide synthase 2 & P35354 & 32 \\
\hline 6 & MMP9 & Matrix metallopeptidase 9 & P50281 & 31 \\
\hline 7 & ESR1 & Estrogen receptor 1 & P03372 & 25 \\
\hline 8 & EP300 & E1A-binding protein $\mathrm{P} 300$ & Q09472 & 23 \\
\hline 9 & TLR4 & Toll-like receptor 4 & O00206 & 23 \\
\hline 10 & PPARG & Peroxisome proliferator-activated receptor gamma & P37231 & 20 \\
\hline 11 & SERPINE1 & Serpin family E member 1 & P05121 & 18 \\
\hline 12 & CDK4 & Cyclin-dependent kinase 4 & $\mathrm{P} 11802$ & 14 \\
\hline 13 & NR3C1 & Nuclear receptor subfamily 3 group C member 1 & P04150 & 14 \\
\hline 14 & CDK1 & Cyclin-dependent kinase 1 & P06493 & 13 \\
\hline 15 & CDK2 & Cyclin-dependent kinase 2 & P24941 & 13 \\
\hline 16 & MMP3 & Matrix metallopeptidase 3 & P08254 & 13 \\
\hline 17 & ADAM17 & ADAM metallopeptidase domain 17 & P78536 & 12 \\
\hline 18 & CHEK1 & Checkpoint kinase 1 & O14757 & 12 \\
\hline 19 & LCK & LCK proto-oncogene, Src family tyrosine kinase & P06239 & 12 \\
\hline 20 & MMP13 & Matrix metallopeptidase 13 & P45452 & 12 \\
\hline 21 & CXCR2 & C-X-C motif chemokine receptor 2 & P25025 & 11 \\
\hline 22 & MMP14 & Matrix metallopeptidase 14 & P50281 & 11 \\
\hline 23 & MMP7 & Matrix metallopeptidase 7 & P09237 & 11 \\
\hline 24 & RPS6KB1 & Ribosomal protein S6 kinase B1 & P23443 & 11 \\
\hline 25 & CA9 & Carbonic anhydrase 9 & Q16790 & 10 \\
\hline 26 & NOS2 & Nitric oxide synthase 2 & P22894 & 10 \\
\hline 27 & PTGS1 & Prostaglandin-endoperoxide synthase 1 & P23219 & 10 \\
\hline 28 & TLR9 & Toll-like receptor 9 & Q9NR96 & 10 \\
\hline 29 & ALOX5 & Arachidonate 5-lipoxygenase & P09917 & 9 \\
\hline 30 & MET & MET proto-oncogene, receptor tyrosine kinase & P08581 & 9 \\
\hline 31 & MMP8 & Matrix metallopeptidase 8 & P22894 & 9 \\
\hline 32 & RAF1 & Raf-1 proto-oncogene, serine/threonine kinase & P04049 & 9 \\
\hline 33 & AURKA & Aurora kinase A & O14965 & 8 \\
\hline 34 & BCL2 & BCL2 apoptosis regulator & P10415 & 8 \\
\hline 35 & ESR2 & Estrogen receptor 2 & Q92731 & 8 \\
\hline 36 & F3 & Coagulation factor III, tissue factor & P13726 & 8 \\
\hline 37 & MIF & Macrophage migration inhibitory factor & P14174 & 8 \\
\hline 38 & NFE2L2 & Nuclear factor, erythroid 2 like 2 & P09237 & 8 \\
\hline 39 & TOP1 & DNA topoisomerase I & P11387 & 8 \\
\hline 40 & ALOX15 & Arachidonate 15-lipoxygenase & P16050 & 7 \\
\hline 41 & DPP4 & Dipeptidyl peptidase 4 & P27487 & 7 \\
\hline 42 & PTPN2 & Protein tyrosine phosphatase nonreceptor type 2 & P17706 & 7 \\
\hline 43 & $\mathrm{ABCC} 1$ & ATP binding cassette subfamily $\mathrm{C}$ member 1 & P33527 & 6 \\
\hline 44 & PPARA & Peroxisome proliferator-activated receptor alpha & Q07869 & 6 \\
\hline 45 & VDR & Vitamin D receptor & P11473 & 6 \\
\hline 46 & CYP2C19 & Cytochrome P450 family 2 subfamily C member 19 & P33261 & 5 \\
\hline 47 & BRAF & B-Raf proto-oncogene, serine/threonine kinase & P15056 & 3 \\
\hline 48 & HTR1A & 5-Hydroxytryptamine receptor $1 \mathrm{~A}$ & P08908 & 3 \\
\hline 49 & RORC & RAR-related orphan receptor C & P51449 & 3 \\
\hline 50 & SLC6A4 & Solute carrier family 6 member 4 & P31645 & 3 \\
\hline 51 & TYR & Tyrosinase & P14679 & 3 \\
\hline 52 & $\mathrm{NR} 1 \mathrm{H} 2$ & Nuclear receptor subfamily 1 group $\mathrm{H}$ member 2 & P55055 & 2 \\
\hline 53 & PTGER2 & Prostaglandin E receptor 2 & P43116 & 2 \\
\hline 54 & HSD11B1 & Hydroxysteroid 11-beta dehydrogenase 1 & P28845 & 2 \\
\hline
\end{tabular}

experimental colitis [30, 31]. Similarly, clinical research studies verified that curcumin inhibits NF- $\kappa \mathrm{B}$ expression by regulating tumor necrosis factor- $\alpha$ (TNF- $\alpha)$ in humans [5]. Further, meta-analyses identified that curcumin have the potential to induce and maintain remission in UC patients with no serious side effects [28].

The 12 found core compounds not only have anti-immune and anti-inflammation effects but also have the effect 


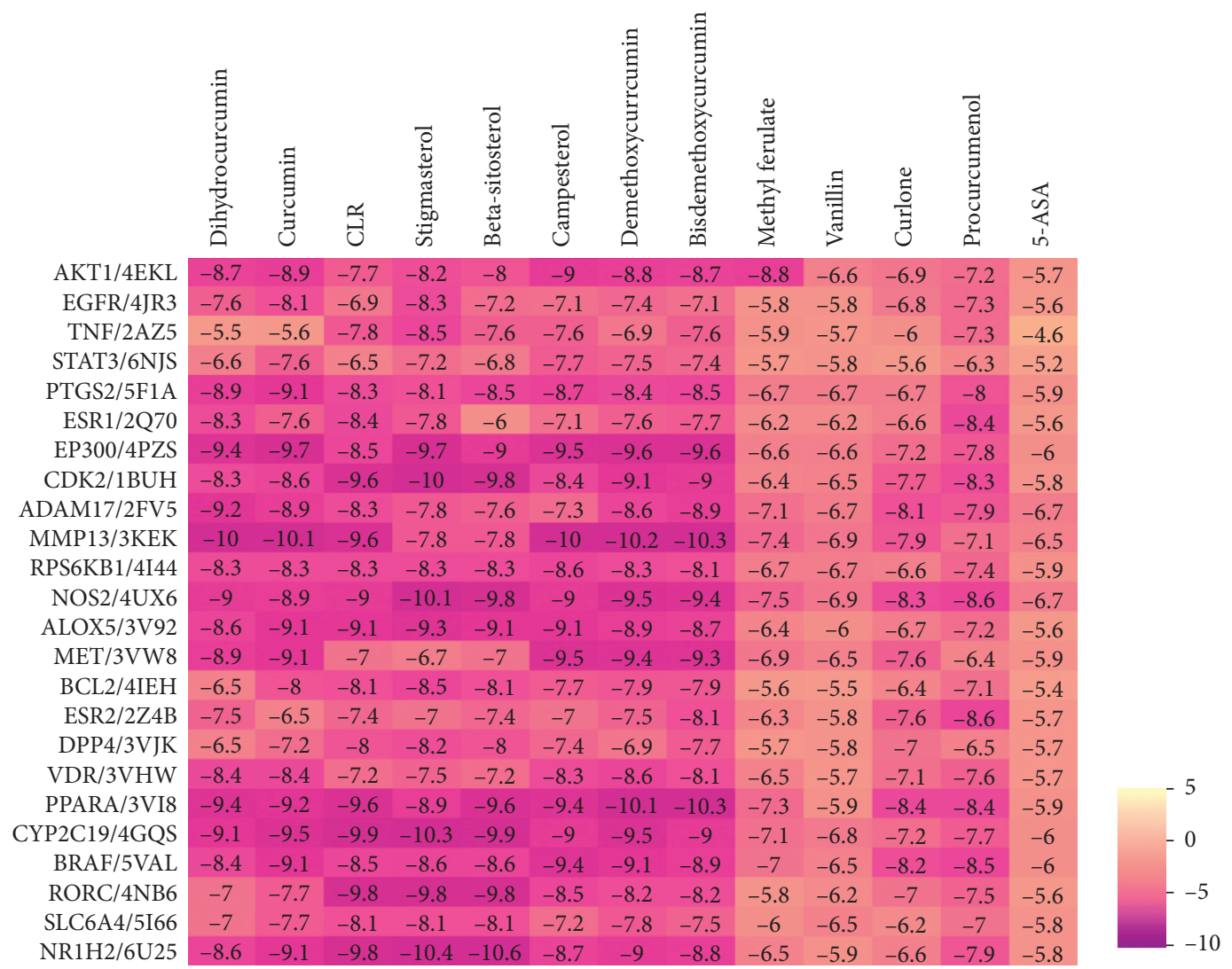

FIGURE 3: 24 proteins whose docking energy with curcuma compounds was above that of 5-ASA.

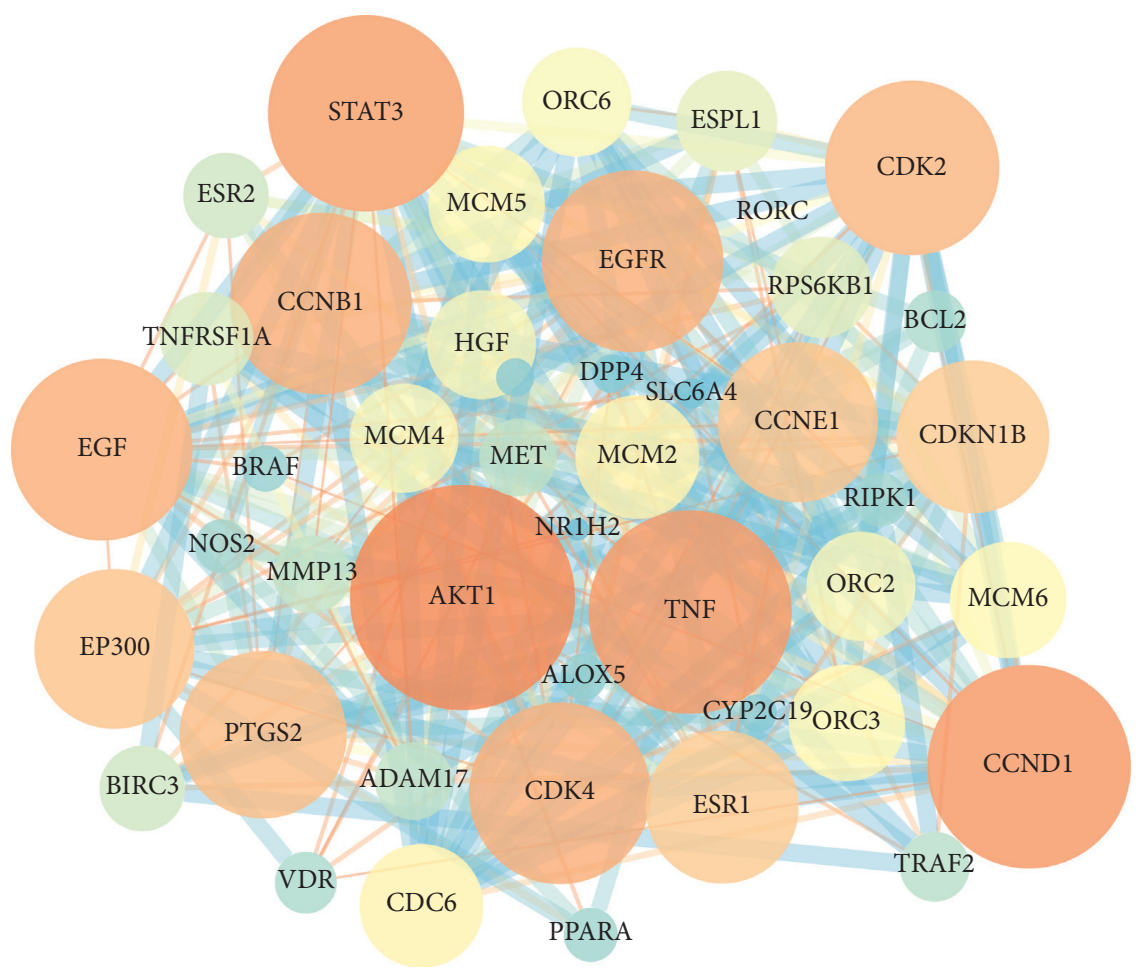

FIgURE 4: PPI network between UC and curcuma. Each node represented a target for curcuma in the treatment of UC. The smaller size and the darker color mean the lower degree value. The edges among nodes display the relationship between different targets. The larger edge size and the brighter edge color mean the higher combination scores. 
Biological process

GO:0071396: cellular response to lipid

GO: 0070482: response to oxygen levels GO:0050727: regulation of inflammatory response GO:0019221: cytokine-mediated signaling pathway

GO: 0048511: rhythmic process

GO:0018105: peptidyl-serine phosphorylation GO:0002237: response to molecule of bacterial origin

GO: 0006979: response to oxidative stress GO: 0062013: positive of small molecule metabolic process

GO: 1901652: response to peptide

GO: 0045787: positive regulation of cell cycle GO: 0080135: regulation of cellular response to stress

GO: 0002521: leukocyte differentiation GO: 0002683: negative regulation of immune system process GO: 0022617: extracellular matrix disassembly

GO: 0031677: response to nutrient levels GO: 0009612: response to mechanical stimulus GO: 0010888: negative regulation of lipid storage

GO: 0009611: responses to wounding GO: 0050673: epithelial cell proliferation

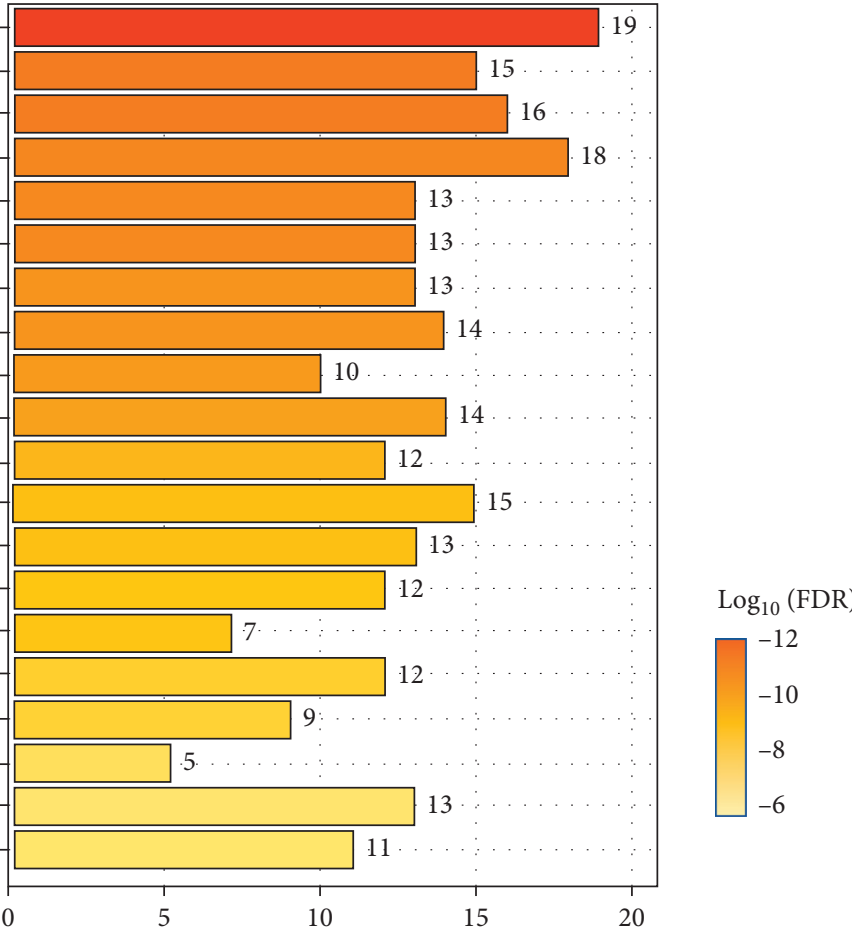

(a)

Molecular function

GO: 0004879: nuclear receptor activity
GO: 0004672: protein kinase activity

GO: 0004222: metalloendopeptidase activity

GO: 0019902: phosphatase binding

GO: 0016705: oxidoreductase activity, acting on paired donors -

GO: 0019904: protein domain specific binding -

GO: 0016702: oxidoreductase activity, acting on single donors

GO: 0002020: protease binding

GO: 0042803: protein homodimerization activity GO: 0004712:protein serine/threonine/tyrosine kinase activity -

GO: 0005178: integrin binding -

GO: 0005126: cytokine receptor binding

GO: 0001618: virus receptor activity GO: 0042562: hormone binding GO: 0016853: isomerase activity

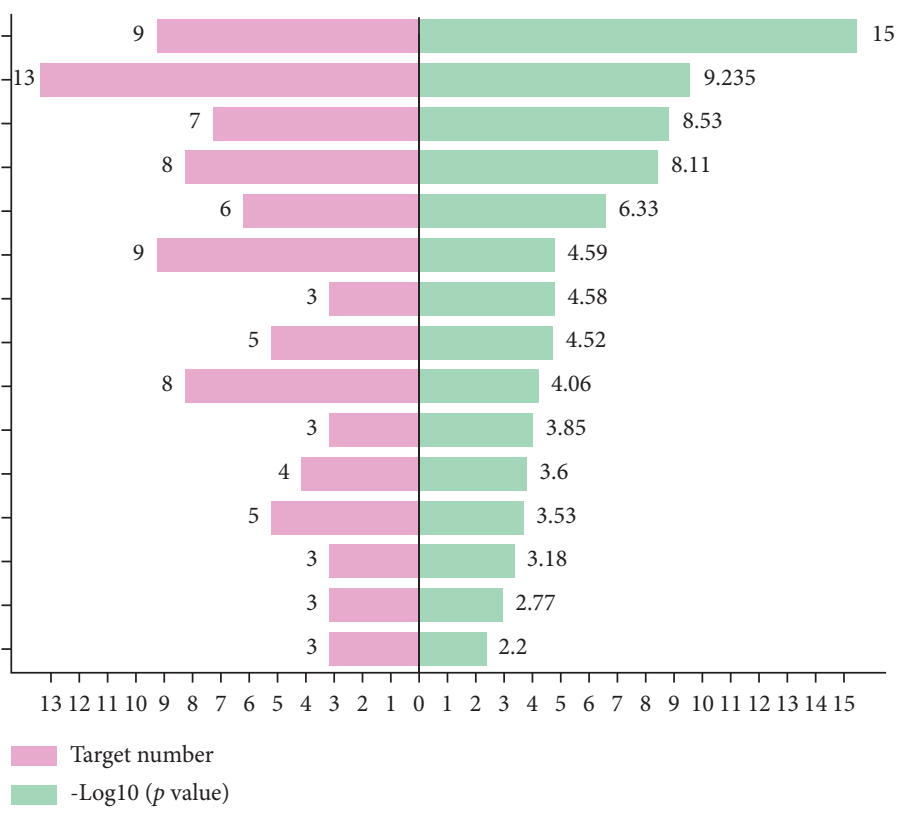

(b)

Figure 5: Continued. 


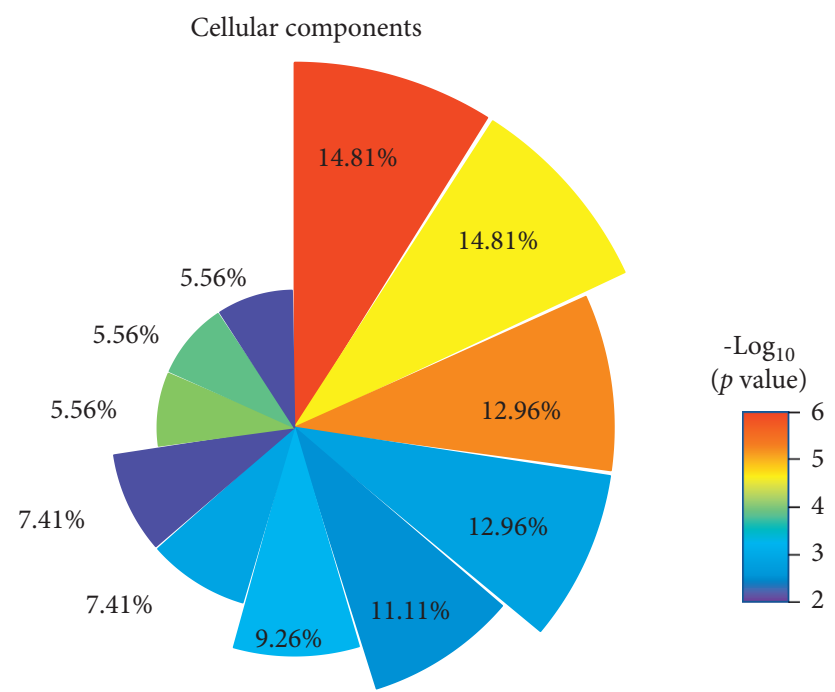

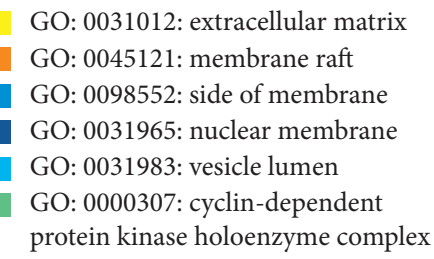

(c)
GO: 0005667: transcription factor complex

GO: 0048471: perinuclear region of cytoplasm

- GO: 0005741: mitochondrial outer membrane

GO: 0032993: protein-DNA complex

GO: 0009925: basal plasma membrane

KEGG pathway gene enrichment

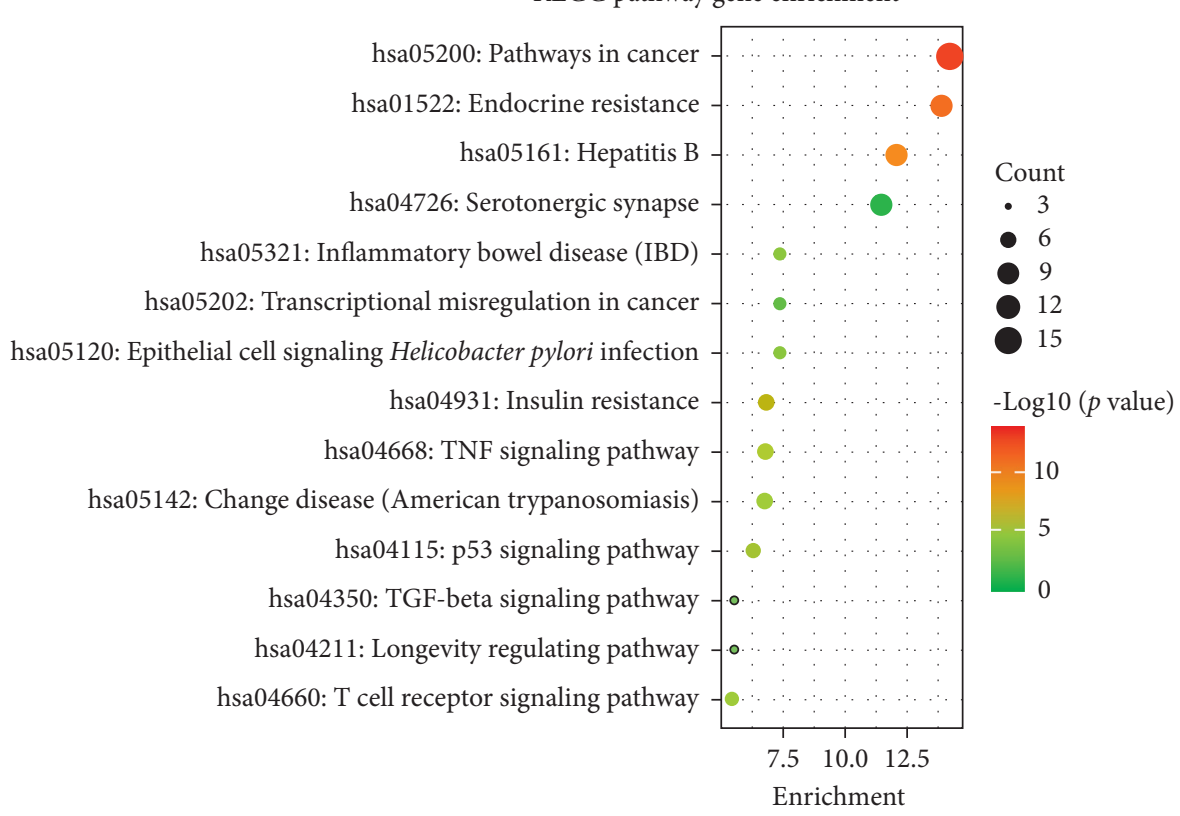

(d)

FIgURe 5: Target biological function and target-pathway analysis. (a) Biological process of curcuma in the treatment of UC. (b) Molecular function of curcuma in the treatment of UC. (c) Cellular components of curcuma in the treatment of UC. (d) Signal pathway of curcuma in the treatment of UC.

of regulating intestinal flora. Scholars verified that the extension of UC is important for the positivity rate of $H$. pylori later [32]. Moreover, it was reported that curcumin downregulated the expression of tumor necrosis factor- $\alpha$ $(\mathrm{TNF}-\alpha)$ through inhibiting NF- $\kappa \mathrm{B}$ expression [32].
Meanwhile, curcumin enhanced the suppressive function of Treg cells and promoted the recovery of damaged colonic mucosa in UC [33]. Previous studies also suggested that CLRs revealed in the research can be included in many immune responses [34]. 


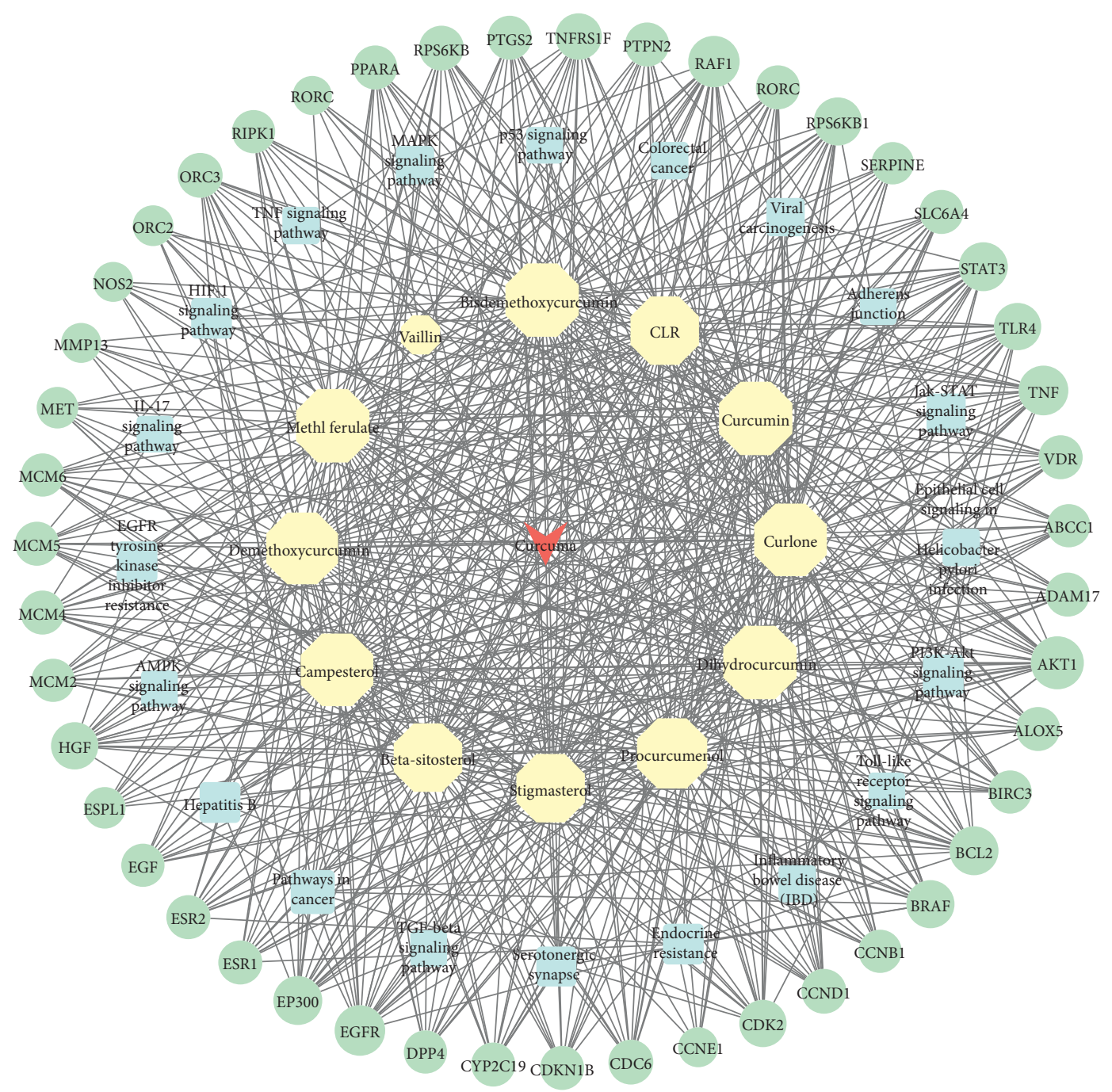

FIgURE 6: Component-target-pathway network of potential pathways in the treatment of UC by curcuma. The green colored circles correspond to the core common targets and the compounds calculated in blue were pathways that contain the targets, and the nodes in yellow are components in curcuma.

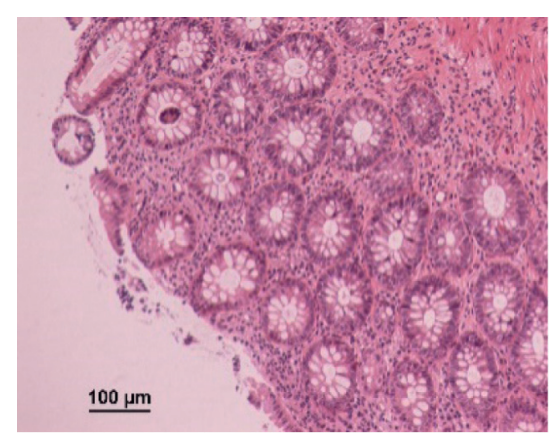

(a)

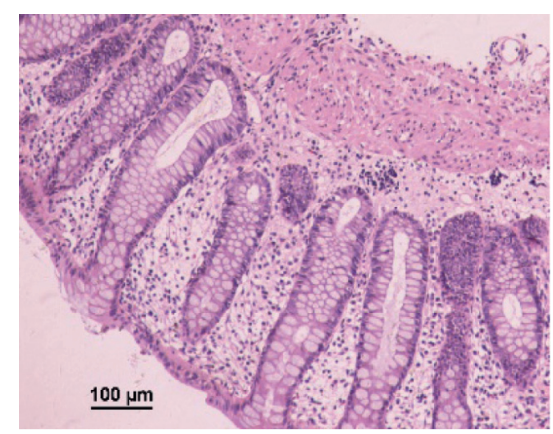

(b)

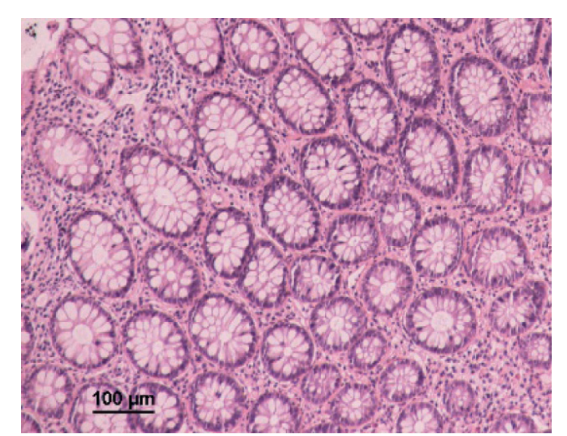

(c)

Figure 7: Effects of curcuma on colon tissue. (a) Control. (b) Model. (c) Curcuma.

Molecular docking verified that the binding energy of 24 key targets was better than that of 5-ASA and lower than -5.5 , meaning the interaction between the compounds and targets of UC can bind tighter than that of 5-ASA, indicating better therapeutic effects. Common target PPI network showed that the targets were cocontrolled by curcuma and UC, which revealed that curcuma could regulate the expression of UC-regulated targets and 


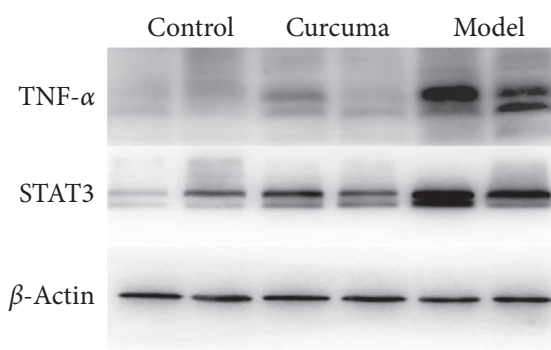

(a)

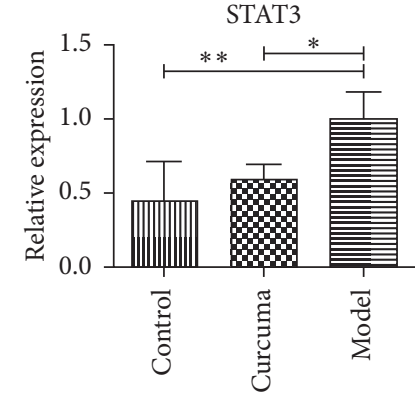

(b)

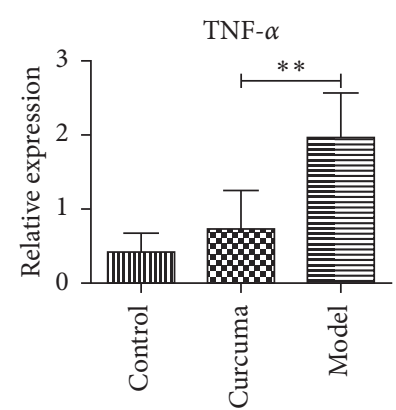

(c)

FIgURE 8: Effects of protein expression in each group $(n=12) .{ }^{*} P<0.05$ and ${ }^{* *} P<0.01$.

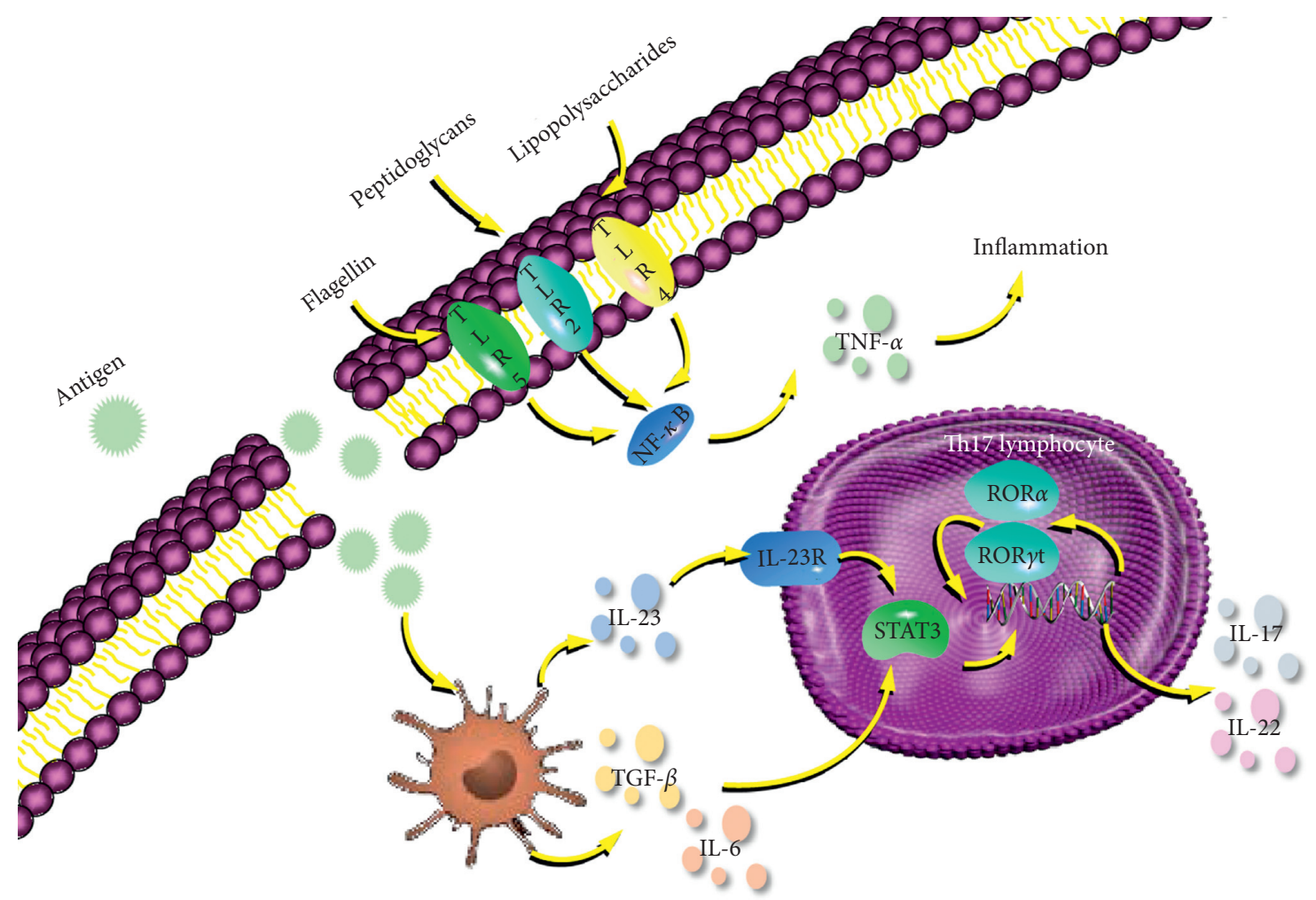

FIGURE 9: Curcuma played a therapeutic role in UC by regulating inflammatory bowel disease pathway.

alleviate UC symptoms. AKT1 (degree=43), EGFR $($ degree $=35)$, TNF- $\alpha($ degree $=35)$, STAT3 $($ degree $=33)$, and PTGS2 (degree $=32$ ) might be the core targets of the PPI network, through whom curcuma may come into play.

In order to predict the mechanism of curcuma in the treatment of UC, we analyzed the key candidate targets by performing GO enrichment results, such as biological processes, molecular functions, and cellular components. The GO terms $(P<0.05)$ indicated that the major hubs were significantly involved in multiple biological processes, including cellular response to lipid, cellular response to organic cyclic compound, and response to steroid hormone. Furthermore, molecular function enrichment analysis showed nuclear receptor activity, transcription factor activity, and steroid hormone receptor activity were contained in the healing process. The active targets involved were AKT1, EGFR, TNF, STAT3, and PTGS2, which mainly concentrate on the molecular processes of immunization, inflammatory reaction, gut microbiota, etc. Some of the genes and mechanisms have been verified in curcumin, while PTGS2 was proposed connecting with the treatment of UC by curcuma firstly as a gene with high possibility. Meanwhile, cellular components consist of RNA polymerase II transcription factor complex, transcription factor complex, nuclear transcription factor complex, and membrane raft, and EGFR, DPP4, ADAM17, BCL2, and CDK2 were contained in the cellular components. The verified impact of EGFR on UC by regulating epithelial barrier function could identify the reliability of this GO analysis [35]. 
The results of pathway analysis and KEGG pathway database [36] analysis showed that the potential mechanisms of curcuma in treating UC were mainly immune regulation, inflammatory reaction, intestinal flora regulation, and the signal pathways related to immunoregulation. Among them, the IBD pathway (Figure 9) was representative and verified in our research.

In the IBD pathway, antigen-presenting cells (APC) endocytosis the invading bacteria and secrete TGF- $\beta$ and IL6. Then, TGF- $\beta$ and IL- 6 stimulate Th17 activating STAT3 with ROR $\gamma$ t and ROR $\alpha$ for further inflammatory cytokines. The activated expression of STAT3 participates in the gene transcription and protein expression of a variety of inflammatory factors such as TNF- $\alpha$ and IL- $1 \beta$, thus promoting the formation and persistent aggravation of inflammation [37-39]. Besides, STAT3, another gene symbol, participates in inflammation. TLR4, an important link in the development of the pathogenesis of UC, can nonspecifically bind to pathogen phase molecules, initiate signal transduction, and eventually lead to the release of nuclear factors [40]. However, lipopolysaccharides activate NK $-\kappa \mathrm{B}$ pathway through TLR4 in intestinal epithelial cells and then induce inflammation by secreting TNF- $\alpha$ in Tolllike receptor signaling pathway. Curcuma may inhibit the inflammation through TLR4, TNF, STAT3, and ROR $\gamma$ t.

\section{Conclusion}

In summary, consistent with clinical experience in the long history, experimental verification, and our HE staining, as well as our WB results, curcuma has significant advantages in the treatment of UC. At the same time, the uncovered targets and pathways were excavated for a better research of how curcuma relieves UC. It has a significant value to provide theoretical basis for clinical treatment of UC and a potent evidence for further study of the mechanism of curcuma in the treatment of UC.

\section{Data Availability}

The data used to support the findings of this study are available from the corresponding author upon request.

\section{Conflicts of Interest}

The authors declare that they have no conflicts of interest.

\section{Authors' Contributions}

SX L conceived and designed the study. SX L and QD L wrote the manuscript. FZ L accomplished statistical data. SX L, QD L, and FZ L gathered active components and target information. FZ L, QD L, and LS L reviewed and edited the manuscript. H C, J L, WC D, and JY S revised the article. All authors read and approved the manuscript.

\section{Acknowledgments}

This research was supported by the National Natural Science Youth Foundation of China (Grant no. 81703986) Professor
RX Z from Tongji University provided valuable suggestions for article revision.

\section{Supplementary Materials}

Table S1: compound targets for each component in curcuma by prediction. Table S2: UC-related targets in GeneCards and DisGeNet and papers published in CNKI and PubMed. Table S3: component-target-pathway connection. (Supplementary Materials)

\section{References}

[1] R. Ungaro, S. Mehandru, P. B. Allen, L. Peyrin-Biroulet, and J.-F. Colombel, "Ulcerative colitis," Lancet (London, England), vol. 389, no. 10080, pp. 1756-1770, 2017.

[2] T. Jess, M. Frisch, and J. Simonsen, "Trends in overall and cause-specific mortality among patients with inflammatory bowel disease from 1982 to 2010," Clinical Gastroenterology and Hepatology, vol. 11, no. 1, pp. 43-48, 2013.

[3] H. S. P. de Souza, C. Fiocchi, and D. Iliopoulos, "The IBD interactome: an integrated view of aetiology, pathogenesis, and therapy," Nature Reviews. Gastroenterology \& Hepatology, vol. 14, no. 12, pp. 739-749, 2017.

[4] R. Mazieiro, R. R. Frizon, S. M. Barbalho, and R. d. A. Goulart, "Is curcumin a possibility to treat inflammatory bowel diseases?" Journal of Medicinal Food, vol. 21, no. 11, pp. 1077-1085, 2018.

[5] M. Simadibrata, C. C. Halimkesuma, and B. M. Suwita, "Efficacy of curcumin as adjuvant therapy to induce or maintain remission in ulcerative colitis patients: an evidencebased clinical review," Acta Medica Indonesiana, vol. 49, no. 4, pp. 363-368, 2017.

[6] Y. Guo, Q. Nie, A.-L. MacLean, Y. Li, J. Lei, and S. Li, "Multiscale modeling of inflammation-induced tumorigenesis reveals competing oncogenic and oncoprotective roles for inflammation," Cancer research, vol. 77, no. 22, pp. 6429-6441, 2017.

[7] S. C. Gupta, S. Patchva, and B. B. Aggarwal, "Therapeutic roles of curcumin: lessons learned from clinical trials," The AAPS Journal, vol. 15, no. 1, pp. 195-218, 2013.

[8] S. Li and B. Zhang, "Traditional Chinese medicine network pharmacology: theory, methodology and application," Chinese Journal of Natural Medicines, vol. 11, no. 2, pp. 110-120, 2013.

[9] J. Huang, F. Cheung, and H.-T. Tan, "Identification of the active compounds and significant pathways of yinchenhao decoction based on network pharmacology," Molecular Medicine Reports, vol. 16, no. 4, pp. 4583-4592, 2017.

[10] A. Daina, O. Michielin, and V. Zoete, "A free web tool to evaluate pharmacokinetics, drug-likeness, and medicinal chemistry friendliness of small molecules," Scientific Reports, vol. 7, no. 1, p. 42717, 2017.

[11] A. Daina, O. Michielin, and V. Zoete, "Swiss target prediction: updated data and new features for efficient prediction of protein targets of small molecules," Nucleic Acids Research, vol. 47, no. 6, pp. W357-W364, 2019.

[12] F. Liu, Q. Zhao, S. Liu et al., "Revealing the pharmacological mechanism of in the treatment of ischemic stroke based on network pharmacology," Evidence-Based Complementary and Alternative Medicine, vol. 2020, Article ID 3236768, 16 pages, 2020. 
[13] S. Paolacci, V. Precone, F. Acquaviva et al., "Genetics of lipedema: new perspectives on genetic research and molecular diagnoses," European Review for Medical and Pharmacological Sciences, vol. 23, no. 13, pp. 5581-5594, 2019.

[14] D. S. Wishart, Y. D. Feunang, A. C. Guo et al., "DrugBank 5.0: a major update to the drugbank database for 2018," Nucleic Acids Research, vol. 46, no. 6, pp. D1074-D1082, 2018.

[15] M.-F. Guo, Y.-J. Dai, J.-R. Gao, and P.-J. Chen, "Uncovering the mechanism of Astragalus membranaceus in the treatment of diabetic nephropathy based on network pharmacology," Journal of Diabetes Research, vol. 2020, Article ID 5947304, 13 pages, 2020.

[16] Y. Zhang, X. Li, X. Xu, and N. Yang, "Mechanisms of paeonia lactiflora in treatment of ulcerative colitis: a network pharmacological study," Medical Science Monitor: International Medical Journal of Experimental and Clinical Research, vol. 25, no. 5, pp. 7574-7580, 2019.

[17] Y. Zhou, B. Zhou, L. Pache et al., "Metascape provides a biologist-oriented resource for the analysis of systems-level datasets," Nature Communications, vol. 10, no. 1, p. 1523, 2019.

[18] X. Lv, "Investigation of the active components and mechanisms of Schisandra chinensis in the treatment of asthma based on a network pharmacology approach and experimental validation," Food \& Function, vol. 11, no. 4, pp. 3032-3042, 2020.

[19] M. Ye, "Network pharmacology, molecular docking integrated surface plasmon resonance technology reveals the mechanism of Toujie Quwen Granules against coronavirus disease 2019 pneumonia," Phytomedicine: International Journal of Phytotherapy and Phytopharmacology, vol. 85, no. 9, p. 153401, 2020.

[20] Long Hua Hospital Shanghai University of Traditional Chinese Medicine, TCM Network Pharmacology Analysis System v1.0[CP/CD].

[21] S. M. D. Rizvi, S. Shakil, and M. Haneef, "A simple click by click protocol to perform docking: AutoDock 4.2 made easy for non-bioinformaticians," EXCLI Journal, vol. 12, no. 2, pp. 831-857, 2013.

[22] A. Cui and Wang, "Chemical constituents from rhizomes of Curcuma longa," Chinese Traditional and Herbal Drugs, vol. 47, no. 7, pp. 1074-1078, 2016, in Chinese.

[23] L. Chen, "Determination of effective constituents in rhizoma curcumae longae," Traditional Chinese Drug Research \& Clinical Pharmacology, vol. 20, no. 3, pp. 253-256, 2009, in Chinese.

[24] D. Gfeller, O. Michielin, and V. Zoete, "Shaping the interaction landscape of bioactive molecules," Bioinformatics (Oxford, England), vol. 29, no. 23, pp. 3073-3079, 2013.

[25] S. C. Gupta, S. Patchva, and B. B. Aggarwal, "Therapeutic roles of curcumin: lessons learned from clinical trials," The AAPS Journal, vol. 15, no. 1, pp. 195-218, 2013.

[26] S. K. Burley, H. M. Berman, G. J. Kleywegt, J. L. Markley, H. Nakamura, and S. Velankar, "Protein data bank (PDB): the single global macromolecular structure archive," Methods in Molecular Biology (Clifton, N.J.), vol. 1607, no. 7, pp. 627-641, 2017.

[27] K.-Y. Hsin, S. Ghosh, and H. Kitano, "Combining machine learning systems and multiple docking simulation packages to improve docking prediction reliability for network pharmacology," PLoS One, vol. 8, no. 12, Article ID e83922, 2013.

[28] N. A. Molodecky, I. S. Soon, D. M. Rabi et al., "Increasing incidence and prevalence of the inflammatory bowel diseases with time, based on systematic review," Gastroenterology, vol. 142, no. 1, pp. 46-54, 2012.

[29] I. Ordás, L. Eckmann, M. Talamini, D. C. Baumgart, and W. J. Sandborn, "Ulcerative colitis," Lancet (London, England), vol. 380, no. 9853, pp. 1606-1619, 2012.

[30] M. S. Baliga, N. Joseph, M. V. Venkataranganna, A. Saxena, V. Ponemone, and R. Fayad, "Curcumin, an active component of turmeric in the prevention and treatment of ulcerative colitis: preclinical and clinical observations," Food \& Function, vol. 3, no. 11, pp. 1109-1117, 2012.

[31] L. Mansour, F. El-Kalla, A. Kobtan et al., "Helicobacter pylori may be an initiating factor in newly diagnosed ulcerative colitis patients: a pilot study," World Journal of Clinical Cases, vol. 6, no. 13, pp. 641-649, 2018.

[32] Y. Wang, Q. Tang, P. Duan, and L. Yang, "Curcumin as a therapeutic agent for blocking NF- $\kappa$ B activation in ulcerative colitis," Immunopharmacology and Immunotoxicology, vol. 40, no. 6, pp. 476-482, 2018.

[33] H.-M. Zhao, R. Xu, X.-Y. Huang et al., "Curcumin improves regulatory $\mathrm{T}$ cells in gut-associated lymphoid tissue of colitis mice," World Journal of Gastroenterology, vol. 22, no. 23, pp. 5374-5383, 2016.

[34] S. C. S. Wolfkamp, M. I. Verstege, E. W. M. Vogels et al., "Single nucleotide polymorphisms in C-type lectin genes, clustered in the IBD2 and IBD6 susceptibility loci, may play a role in the pathogenesis of inflammatory bowel diseases," European Journal of Gastroenterology \& Hepatology, vol. 24, no. 8, pp. 965-970, 2012.

[35] M. Shimodaa, K. Horiuchib, A. Sasaki et al., "Epithelial cellderived a disintegrin and metalloproteinase-17 confers resistance to colonic inflammation through EGFR activation," EBioMedicine, vol. 5, no. 1, pp. 114-116, 2016.

[36] M. Tanabe and M. Kanehisa, "Using the KEGG database resource," Current Protocols in Bioinformatics, vol. 15, no. 5, 2012.

[37] J. Mudter and M. F. Neurath, "Il-6 signaling in inflammatory bowel disease: pathophysiological role and clinical relevance," Inflammatory Bowel Diseases, vol. 13, no. 8, pp. 1016-1023, 2007.

[38] P. Amadio, E. Tarantino, L. Sandrini, E. Tremoli, and S. S. Barbieri, "Prostaglandin-endoperoxide synthase-2 deletion affects the natural trafficking of Annexin A2 in monocytes and favors venous thrombosis in mice," Thrombosis and Haemostasis, vol. 117, no. 8, pp. 1486-1497, 2017.

[39] L. Wang-Bishop, Z. Chen, A. Gomaa et al., "Inhibition of AURKA reduces proliferation and survival of gastrointestinal cancer cells with activated KRAS by preventing activation of RPS6KB1," Gastroenterology, vol. 156, no. 3, pp. 662.e7-675.e7, 2019.

[40] J. Epstein, G. Docena, T. T. MacDonald, and I. R. Sanderson, "Curcumin suppresses p38 mitogen-activated protein kinase activation, reduces IL-1beta and matrix metalloproteinase- 3 and enhances IL-10 in the mucosa of children and adults with inflammatory bowel disease," The British Journal of Nutrition, vol. 103, no. 6, pp. 824-832, 2010. 